\title{
When the telegrapher's equation furnishes a better approximation to the transport equation than the diffusion approximation
}

\author{
Josep M. Porrà and Jaume Masoliver \\ Departament de Física Fonamental, Universitat de Barcelona, Diagonal 647, 08028-Barcelona, Spain \\ George H. Weiss \\ Division of Computer Research and Technology, National Institutes of Health, Bethesda, Maryland 20892
}

(Received 8 July 1996)

\begin{abstract}
It has been suggested that a solution to the transport equation which includes anisotropic scattering can be approximated by the solution to a telegrapher's equation [A.J. Ishimaru, Appl. Opt. 28, 2210 (1989)]. We show that in one dimension the telegrapher's equation furnishes an exact solution to the transport equation. In two dimensions, we show that, since the solution can become negative, the telegrapher's equation will not furnish a usable approximation. A comparison between simulated data in three dimensions indicates that the solution to the telegrapher's equation is a good approximation to that of the full transport equation at the times at which the diffusion equation furnishes an equally good approximation. [S1063-651X(97)04205-0]
\end{abstract}

PACS number(s): $05.60 .+\mathrm{w}$

\section{INTRODUCTION}

Problems related to the transport of matter in disordered media are common to a number of areas of science and technology, and have given rise to a considerable amount of research aimed at delineating properties of motion in such media $[1,2]$. When the medium is a continuum, a natural starting point for any analysis is based on solving an appropriate transport equation. Since there are no general solutions other than purely numerical ones for such equations it is often difficult to fit experimental data to theory, as would be necessary to determine physically significant parameters. Hence a number of approximation schemes have been used to derive more tractable mathematical models.

The simplest such model which is adequate in many applications approximates the full transport equation by a diffusion equation. A related model which has recently been applied to problems arising in tissue optics is based on the theory of lattice random walks [3-5]. Both of these are deficient in failing to properly account for anisotropic scattering, although this problem tends to vanish at increasingly long times, which is essentially the regime in which the central limit theorem is equivalent to the diffusion approximation. Nevertheless the diffusion equation is appealing because it is trivial to solve in unbounded spaces and easy to specify boundary conditions associated with finite media.

Several compromises have been suggested to incorporate at least some aspects of anisotropic scattering into the diffusion picture. A strategy used in several optical applications is to correct the diffusion constant by using a characteristic parameter related to the degree of anisotropic scattering [6]. A recent suggestion has been made by Ishimaru in the context of tissue optics, that anisotropy can be incorporated into the analysis by replacing the diffusion equation by a telegrapher's equation (TE) [7], which has the form

$$
\left(\partial^{2} P / \partial t^{2}\right)+[(1 / T)(\partial P / \partial t)]=c^{2} \nabla^{2} P
$$

where $T$ is a parameter with the dimensions of time and $c$ is a speed. Two motivating factors for Ishimaru's suggestion are to be found in an observation by Goldstein that the continuum limit of a persistent random walk is a telegrapher's equation [8], and from an approximation based on the transport equation derived by Ishimaru [9].

In the present paper we investigate whether the TE provides a better approximation to the solution of the full transport equation over a physically relevant time span than does the diffusion equation. We show that this is true for the single-speed model in one dimension, but that in two dimensions the TE has a solution that is negative in some region of the plane, and therefore cannot be identified as a probability density. In the case of three dimensions we show, by comparing the theoretical results to data simulated using a physically plausible model, that the solution to the TE is not a good approximation to the simulated results except at comparatively long times, at which the solution of the TE essentially coincides with the solution to the diffusion equation.

\section{TRANSPORT EQUATION}

In modeling transport we will consider the single-speed model only [10]. Let $\lambda$ be the scattering rate. Absorption in the medium will be modeled in terms of Beer's law characterized by a rate parameter $\mu$ [5]. Only the case of a pulse propagating in a translationally invariant medium will be considered. Let the direction in which a single particle moves be denoted by the solid angle $\boldsymbol{\Omega}$. In three dimensions $\boldsymbol{\Omega}=(\theta, \varphi)$, where $\theta$ is the polar angle and $\varphi$ is the azimuthal angle. Further, let $\beta\left(\boldsymbol{\Omega} \mid \mathbf{\Omega}^{\prime}\right)$ denote the scattering kernel, i.e., the probability that a single scattering changes the direction of the photon path from $\boldsymbol{\Omega}^{\prime}$ to a direction falling somewhere in the infinitesimal interval $(\boldsymbol{\Omega}, \boldsymbol{\Omega}+d \boldsymbol{\Omega})$ is equal to $\beta\left(\boldsymbol{\Omega} \mid \boldsymbol{\Omega}^{\prime}\right) d \boldsymbol{\Omega}$. The speed between collisions will be taken to be a constant $v$, and the corresponding velocity, which takes the direction into account, will be denoted by $v(\boldsymbol{\Omega})$. For example, in three dimensions

$$
v(\boldsymbol{\Omega})=(v \sin \theta \cos \varphi, v \sin \theta \sin \varphi, v \cos \theta),
$$

where $\theta$ and $\varphi$ are the polar and azimuthal angles, respectively. The object of our investigation is to find the probabil- 
ity density for the particle to be at $\boldsymbol{r}$ moving in the direction $\boldsymbol{\Omega}$ at time $t$. This function will be denoted by $p(\boldsymbol{r}, \mathbf{\Omega}, t)$. In this notation the full transport equation is

$$
\begin{aligned}
\partial p / \partial t= & -(\lambda+\mu) p-v(\boldsymbol{\Omega}) \cdot \boldsymbol{\nabla} p \\
& +\lambda \int p\left(\boldsymbol{r}, \boldsymbol{\Omega}^{\prime}, t\right) \beta\left(\boldsymbol{\Omega} \mid \mathbf{\Omega}^{\prime}\right) d \mathbf{\Omega}^{\prime} .
\end{aligned}
$$

Only the case in which the initial condition is a single pulse will be analyzed. In three dimensions this allows us to write

$$
p\left(\boldsymbol{r}, \boldsymbol{\Omega}^{\prime}, 0\right)=(1 / 4 \pi) \delta(\boldsymbol{r})=\delta(r) / 16 \pi^{2} r^{2} .
$$

We will assume that the kernel $\beta\left(\boldsymbol{\Omega} \mid \boldsymbol{\Omega}^{\prime}\right)$ depends only on the deflection angle $\gamma$, i.e., the angle between directions $\boldsymbol{\Omega}$ and $\boldsymbol{\Omega}^{\prime}$. In this case, the deviation from isotropic scattering will be denoted by $g=\langle\cos \gamma\rangle$. The solution to Eq. (2) can be expanded in terms of spherical harmonics, although in practice the series is generally truncated. The so-called $P_{1}$ approximation, commonly used in nuclear reactor analysis $[10,11]$, is equivalent to the assumption that $p(\boldsymbol{r}, \boldsymbol{\Omega}, t)$ is only weakly dependent on angle. In three dimensions this is equivalent to writing [11]

$$
p(\boldsymbol{r}, \boldsymbol{\Omega}, t) \approx(1 / 4 \pi) P(\boldsymbol{r}, t)+\left(3 / 4 \pi v^{2}\right) v(\boldsymbol{\Omega}) \cdot \boldsymbol{J}(\boldsymbol{r}, t) .
$$

Here $P(\boldsymbol{r}, t)$ is the angle-averaged value of $p(\boldsymbol{r}, \boldsymbol{\Omega}, t)$, i.e.,

$$
P(\boldsymbol{r}, t)=\int p(\boldsymbol{r}, \boldsymbol{\Omega}, t) d \mathbf{\Omega},
$$

and the flux $\boldsymbol{J}(\boldsymbol{r}, t)$ is related to $p(\boldsymbol{r}, \boldsymbol{\Omega}, t)$ by

$$
\boldsymbol{J}(\boldsymbol{r}, t)=\int v(\boldsymbol{\Omega}) p(\boldsymbol{r}, \boldsymbol{\Omega}, t) d \boldsymbol{\Omega} .
$$

The angle variables appear linearly in Eq. (4) as indicated. On substituting this expansion into Eq. (2), we find a coupled set of equations for $P(\boldsymbol{r}, t)$ and $\boldsymbol{J}(\boldsymbol{r}, t)$, which is

$$
\begin{gathered}
\partial P / \partial t=-\mu P-\boldsymbol{\nabla} \cdot \boldsymbol{J}, \\
\partial \boldsymbol{J} / \partial t=-\left(\lambda^{\prime}+\mu\right) \boldsymbol{J}-\left(v^{2} / 3\right) \nabla P,
\end{gathered}
$$

where

$$
\lambda^{\prime}=\lambda(1-g)
$$

is an anisotropy-corrected scattering rate. On the further assumption that $\partial \boldsymbol{J} / \partial t$ can be neglected in comparison to the terms on the right-hand side of Eq. (6b), one finds a damped diffusion equation for $P(\boldsymbol{r}, t)$,

$$
(\partial P / \partial t)+\mu P=D \nabla^{2} P,
$$

with an effective diffusion constant

$$
D=v^{2} /\left[3\left(\lambda^{\prime}+\mu\right)\right] .
$$

If the time derivative of the flux is not considered negligible then the function $\boldsymbol{J}(\boldsymbol{r}, t)$ can be eliminated from Eq. (6b), and the equation satisfied by $P(r, t)$ is the damped telegrapher's equation

$$
\frac{\partial^{2} P}{\partial t^{2}}+\left(\lambda^{\prime}+2 \mu\right) \frac{\partial P}{\partial t}+\mu\left(\lambda^{\prime}+\mu\right) P=c^{2} \nabla^{2} P,
$$

where the velocity $c$ is equal to $v / \sqrt{3}$. This equation has been obtained by taking the divergence on both sides of Eq. (6b), commuting the divergence operator and $\partial / \partial t$ on the left-hand side, and finally using Eq. (6a) to write $\boldsymbol{\nabla} \cdot \boldsymbol{J}$ in terms of $P$.

We note that the substitution $P(\boldsymbol{r}, t)=Q(\boldsymbol{r}, t) e^{-\mu t}$ transforms Eq. (8) into the standard form of the TE in Eq. (1) with the time parameter $T=1 / \lambda^{\prime}$. We also observe that the solution to the telegrapher's equation at very long times reduces to a Gaussian [12], which is the solution to the diffusion equation in free space. The question to be addressed is whether the solution of the telegrapher's equation more faithfully reproduces the solution of the full transport equation than does the diffusion equation. We consider this problem in one, two, and three dimensions.

\section{A. One dimension}

In one dimension a particle can move in only two directions, the coordinate of its location at any time $t$ either increasing or decreasing with a constant speed. Thus a description of the system requires consideration of the evolution of two probability densities, $p_{i}(x, t), i= \pm 1$, where $i= \pm 1$ refers to a particle having a speed $\pm v$. If $P(x, t)$ and $J(x, t)$ are defined by

$$
\begin{gathered}
P(x, t)=p_{1}(x, t)+p_{-1}(x, t), \\
J(x, t)=v\left[p_{1}(x, t)-p_{-1}(x, t)\right],
\end{gathered}
$$

then the full transport equation coincides exactly with the $P_{1}$ approximation. Hence it follows from our earlier remark that the equation satisfied by $P(x, t)$ is the damped telegrapher's equation, Eq. (8), with $c=v$. Hence in one dimension the TE provides an exact, rather than approximate, solution to the full transport equation. This is also implied in the work of Goldstein [8].

\section{B. Two dimensions}

The problem of the appropriateness of the TE as a model accounting for anisotropy is much more interesting in dimensions greater than one, since the $P_{1}$ approximation is not equivalent to the full transport equation. In two dimensions the angular coordinate $\Omega$ consists of a single polar angle $\theta$. The $P_{1}$ approximation is found by retaining only the terms $n=-1,0$, and 1 in the Fourier expansion of $p(r, \theta, t)$,

$$
p(\boldsymbol{r}, \theta, t)=\sum_{n=-\infty}^{\infty} b_{n}(\boldsymbol{r}, t) e^{i n \theta},
$$

and the telegrapher's equation is given by Eq. (8) with $c=v / \sqrt{2}$. We have shown that a simple transformation of the dependent variable serves to eliminate the parameter $\mu$, allowing us to simplify the analysis by neglecting absorption. The solution to the TE in two dimensions for the set of isotropic initial conditions

$$
p(\boldsymbol{r}, 0)=\delta(\boldsymbol{r}), \quad \partial p /\left.\partial t\right|_{t=0}=0,
$$

can be obtained by Laplace-Fourier transform, and is written in terms of the dimensionless combination

$$
\alpha=\left(\lambda^{\prime} / v \sqrt{2}\right) \sqrt{v^{2} t^{2} / 2-r^{2}}
$$



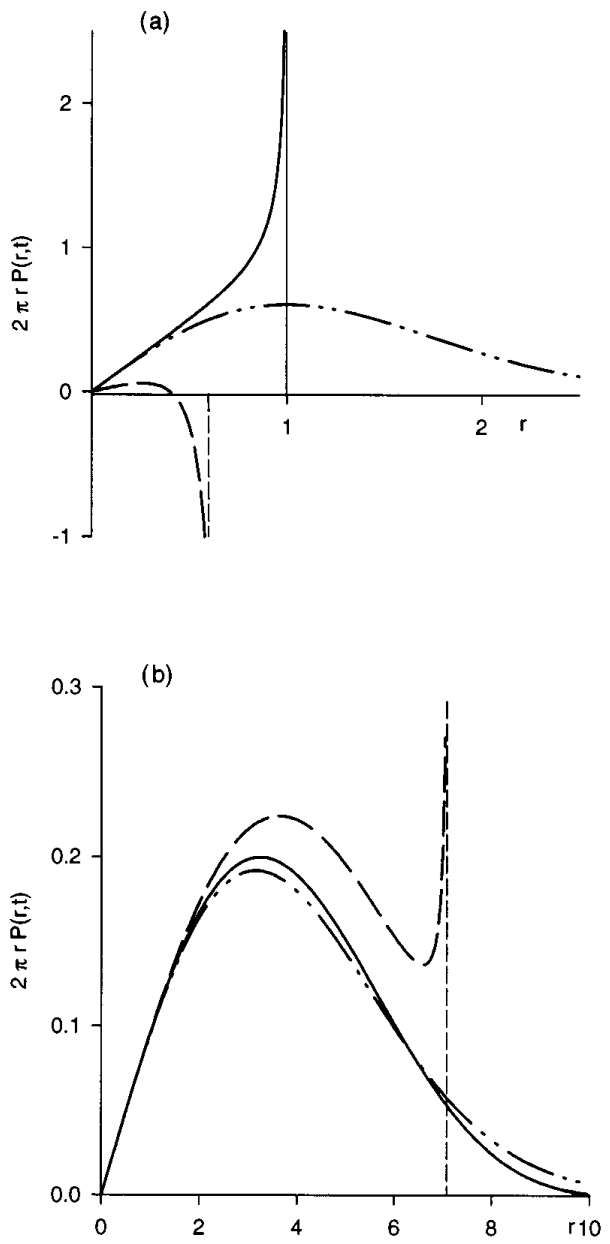

FIG. 1. The two-dimensional radial probability densities $2 \pi r P_{\text {exact }}(r, t) \quad$ (solid line), $2 \pi r P_{\mathrm{TE}}(r, t)$ (dashed line), and $2 \pi r P_{\text {diffusion }}(r, t)$ (dot-dashed line) plotted as a function of $r$ for (a) $t=1$ and (b) $t=10$. Both sets of curves were generated using the parameter values $\lambda=v=1$.

as

$$
\begin{aligned}
P(r, t)= & \frac{\lambda^{\prime} e^{-\lambda^{\prime} t / 2}}{2^{3 / 2} \pi v \sqrt{v^{2} t^{2} / 2-r^{2}}}\left(\cosh \alpha+\frac{\lambda^{\prime} t \sinh \alpha}{2 \alpha}\right) \\
& \times H(v t / \sqrt{2}-r)+\frac{e^{-\lambda^{\prime} t / 2}}{\pi \sqrt{2} v} \cosh \alpha \\
& \times \frac{\partial}{\partial t}\left[\frac{H(v t / \sqrt{2}-r)}{\sqrt{v^{2} t^{2} / 2-r^{2}}}\right] .
\end{aligned}
$$

In this equation $H(z)$ is the Heaviside step function, $H(z)=1, z>0$, and $H(z)=0$ for $z<0$.

Figure 1 compares profiles of the angular density $2 \pi r P(r, t)$ for the isotropic model where $g=0$. The three values of $P(r, t)$ that are plotted are $P_{\text {exact }}(r, t)$, the exact solution, $P_{\mathrm{TE}}(r, t)$, the solution obtained from Eq. (13); and $P_{\text {diffusion }}(r, t)$, the solution obtained from the diffusion approximation. The exact solution was obtained in [13] and reads

$$
\begin{aligned}
P_{\text {exact }}(\boldsymbol{r}, t)= & e^{-\lambda t}\left[\frac{\delta(r-v t)}{2 \pi r}+\frac{\lambda}{2 \pi v \sqrt{v^{2} t^{2}-r^{2}}}\right. \\
& \left.\times \exp \left(\frac{\lambda \sqrt{v^{2} t^{2}-r^{2}}}{v}\right) H(v t-r)\right] .
\end{aligned}
$$

Note that the exact result, Eq. (14), and the solution to TE, Eq. (13), both converge when $t \rightarrow \infty$ to the diffusion approximation given by

$$
P_{\text {diffusion }}(\boldsymbol{r}, t)=\left(\lambda / 2 v^{2} \pi t\right) \exp \left(-\lambda r^{2} / 2 v^{2} t\right) .
$$

We note that the divergence of $P(r, t)$ at $r=v t / \sqrt{2}$ [c.f. Eq. (13) is due to the time propagation of the singular initial condition Eq. (11). The same happens to $P_{\text {exact }}(r, t)$ at the boundary $r=v t$. We also observe that the divergence is integrable, and both $P(r, t)$ and $P_{\text {exact }}(r, t)$ are normalized to 1 .

It is evident that the solution for $P_{\mathrm{TE}}(r, t)$ becomes negative somewhere in the interval $(0, v t / \sqrt{2})$, and therefore cannot be a probability density. Such behavior is a characteristic of solutions found in even-dimensional spaces [12]. In consequence, the TE cannot furnish an accurate approximation to the solution of the full transport equation in even dimensions, except in the limit of times so large that the solution of the TE is essentially equal to the solution provided by the diffusion equation. In addition, even in the regions where the solution of the TE is positive, numerical data indicate that the diffusion approximation is better than the TE solution (see Fig. 1).

\section{Three dimensions}

An expression is known for the Fourier-Laplace transform of the solution of the isotropic transport equation in Eq. (2) for the fully isotropic case [13],

$$
\beta\left(\boldsymbol{\Omega} \mid \mathbf{\Omega}^{\prime}\right)=1 / 4 \pi .
$$

Since the transform cannot be inverted except numerically, we conducted a simulation study to test the quality of the approximation furnished by the TE. Our simulations were only for particles moving in an unbounded space. The time between two successive scattering events were random variables whose properties were described by a negative exponential probability density

$$
\psi(t)=\lambda e^{-\lambda t}
$$

In our choice of the form of the kernel $\beta\left(\boldsymbol{\Omega} \mid \boldsymbol{\Omega}^{\prime}\right)$, we assumed that this depended only on the deflection angle $\gamma$, while the azimuthal angle $\varphi$ was assumed to be uniformly distributed in the interval $(-\pi, \pi)$. Between scatterings the particles were assumed to move in straight lines. The effect of anisotropic scattering was mimicked by choosing the scattering angle according to the widely used Henyey-Greenstein phase function [14]

$$
F(\gamma)=\frac{1-g^{2}}{2 g}\left[\frac{1}{1-g}-\frac{1}{\sqrt{1+g^{2}-2 g \cos \gamma}}\right], \quad 0 \leqslant \gamma \leqslant \pi .
$$


Other phase functions may have been used, because the only requirement is that the anisotropic factor $g=\langle\cos \gamma\rangle \neq 0$ is different than zero.

The data generated in this way were compared with results generated from the solution to the three-dimensional TE in free space. Analogously to the two-dimensional case, this solution is expressed in terms of the variable

$$
\xi=\left(\lambda^{\prime} / 2 v\right) \sqrt{v^{2} t^{2}-3 r^{2}}
$$

as

$$
\begin{aligned}
P(\boldsymbol{r}, t)= & \frac{e^{-\lambda^{\prime} t / 2}}{2 \pi r}\left[\frac{\sqrt{3} \lambda^{\prime}}{4 v}\left(1+\frac{\lambda^{\prime} t}{4}\right) \delta(r-v t / \sqrt{3})\right. \\
& \left.-\frac{1}{2} \frac{\partial}{\partial r} \delta(r-v t / \sqrt{3})\right]+\frac{3 \lambda^{\prime} e^{-\lambda^{\prime} t / 2}}{16 \pi v^{2} \sqrt{v^{2} t^{2} / 3-r^{2}}} \\
& \times\left[I_{1}(\xi)+\frac{\lambda^{\prime} t}{2 \xi} I_{2}(\xi)\right] H(v t / \sqrt{3}-r),
\end{aligned}
$$

where $I_{n}(z)$ are modified Bessel functions. At long times the solution given in Eq. (20) tends toward the Gaussian solution of the diffusion equation, i.e.,

$$
P(\boldsymbol{r}, t) \rightarrow\left(3 \lambda^{\prime} / 4 \pi v^{2} t\right)^{3 / 2} \exp \left(-3 \lambda^{\prime} r^{2} / 4 v^{2} t\right) .
$$

Figure 2 shows the results of $10^{5}$ simulated runs as described above and carried out for the parameter values $g=0.5$, $v=1$, and $\lambda=1$. Two sets of data are shown that correspond to times $t=5$ and 25, which is in the regime in which the Gaussian form in Eq. (21) is expected to be valid. The agreement with the asymptotic Gaussian form of $P(\boldsymbol{r}, t)$ at the larger time is seen to be quite good, while the agreement with the TE at shorter times is not.

The solution given in Eq. (20) is non-negative, but the fact that the rescaled speed of propagation cannot correspond to the true speed of the scattered particles prevents finding a more accurate approximation, at least in terms of the TE. This is apparently due to the contributions to $P(\boldsymbol{r}, t)$ from particles which have never been scattered. It is possible that this shortcoming can be overcome by explicitly decomposing the solution of the transport equation into a contribution from the unscattered particles, and one from those that were scattered at least once, as is sometimes done in other problems related to photon diffusion in turbid media [7]. A study of this type of approximation is presently under consideration.
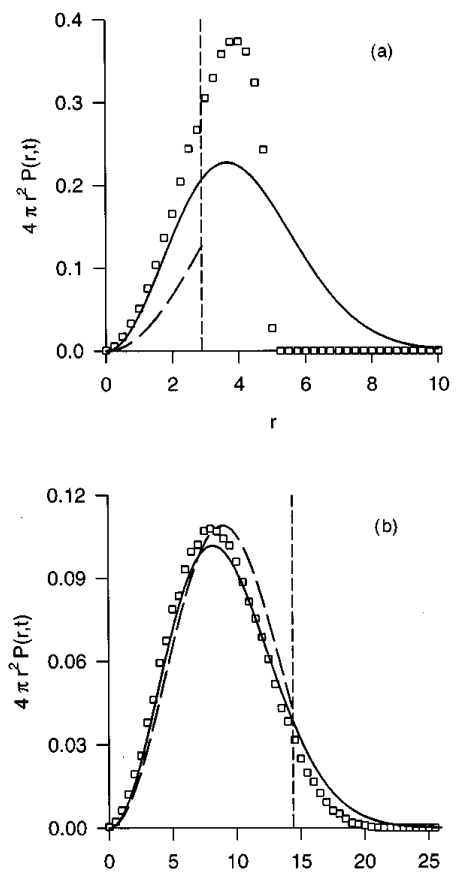

FIG. 2. A comparison of simulation results (square symbols), the solution to the three-dimensional TE (dashed line), and the diffusion approximation (solid line) plotted as a function of $r$ for (a) $t=5$ and (b) $t=25$. The curves and data points in these curves were generated using parameter values $\lambda=v=1$ and the asymmetry factor $g=0.5$. The curves at the earlier time agree poorly with solutions to both the TE and diffusion equations, while those at the later time agree reasonably well with the solution to the $\mathrm{TE}$, but that agrees well with the solution to the diffusion equation.

\section{A FINAL COMMENT}

Both simulations and analytical considerations suggest that, except in one dimension, there are significant difficulties in basing an approximate solution to the full transport equation on an "equivalent" telegrapher's equation. In two dimensions this occurs because the solution to the telegrapher's equation has regions in which the solution is negative. In three dimensions the difficulty can be traced to an inaccurate accounting for particles that remain unscattered at time $t$. At very long times, when the number of such particles is considerably reduced, the solution to the telegrapher's equation is quite accurately approximated by the solution to a diffusion equation. Our simulations suggest that in this regime the solution to the full transport equation can also be modeled quite accurately.
[1] Photon Migration in Tissues, edited by B. Chance (Plenum, New York, 1989).

[2] Laser-Doppler Flowmetry, edited by A. P. Shepherd, Jr. and P. A. Öberg (Kluwer, Boston, 1990).

[3] R. F. Bonner et al., J. Opt. Soc. Am. A 4, 423 (1987).

[4] G. H. Weiss, Aspects and Applications of the Random Walk (NorthHolland, Amsterdam, 1994).

[5] A. H. Gandjbakhche and G. H. Weiss, Prog. Opt. 34, 333 (1995).

[6] A. J. Ishimaru, Wave Propagation and Scattering in Random Media (Academic, New York, 1978).

[7] A. J. Ishimaru, Appl. Opt. 28, 2210 (1989).
[8] S. Goldstein, Q. J. Mech. Appl. Math. 4, 129 (1951).

[9] A. J. Ishimaru, J. Opt. Soc. Am. 68, 1045 (1978).

[10] J. J. Duderstadt and L. J. Hamilton, Nuclear Reactor Analysis (Wiley, New York, 1976).

[11] J. J. Duderstadt and W. R. Martin, Transport Theory (Wiley, New York, 1979).

[12] P. M. Morse and H. Feshbach, Methods of Theoretical Physics (McGraw-Hill, New York, 1953).

[13] J. Masoliver et al., Physica A 193, 469 (1993).

[14] L. G. Henyey and J. L. Greenstein, Astrophys. J. 93, 70 (1941). 